\title{
A transient boundary element method for acoustic scattering from mixed regular and thin rigid bodies
}

\section{Abstract}

Boundary Element Methods (BEMs) may be used to predict the scattering of sound by obstacles, which has accelerated the prototyping of new room acoustic treatments such as diffusers. Unlike the more popular frequency domain method, the time domain BEM is usually solved in an iterative manner which means it can exhibit instability, a crucial impediment to its widespread use. These instabilities are primarily associated with the resonance of cavities formed by closed surface sections, but may also be caused by discretisation or integration error corrupting physical damped resonances.

Regular BEM implementations cannot model objects with thin sections due to a phenomenon known as Thin Shape Breakdown. This paper develops an algorithm which combines an accepted approach for modelling thin plates with the Combined Field Integral Equation which eradicates cavity resonances, thereby permitting models of mixed regular and thin bodies. Accuracy and stability are tested by comparison to verified frequency domain BEMs, examination of the transient response, and pole decomposition. This is done for a simple obstacle and a Schroeder diffuser, which comprises a series of wells separated by thin fins. The approach is successful but universal stability cannot be guaranteed for the diffuser. It is suggested that instability is caused by the lightly damped resonances of the wells being corrupted into divergent behaviour by numerical errors. 
PACS: 43.20.Fn, 43.20.Px, 43.55.Br

\section{Introduction}

Scattering of sound by an obstacle is a fundamental process in acoustics and predicting it accurately a powerful tool in the design of acoustic treatments. A Boundary Element Method (BEM) model may be used to calculate such scattering by considering only the boundaries between obstacles and air, as it is known how sound travels unobstructed. This produces smaller, simpler meshes compared to volumetric methods such as finite element and finite difference time domain (FDTD). It also permits an unbounded volume of air to be modelled, making it ideal for free-field scattering scenarios.

Most BEMs assume harmonic excitation so the unknowns are time invariant and complex. Whilst this frequency domain analyses is a useful tool, the transient behaviour witnessed in the real world may only be recovered by solving many frequency domain models and then applying an inverse discrete Fourier transform. An alternative is to drop the time invariant assumption and formulate the BEM in the time domain as is presented herein. This approach was first published by Friedman and Shaw in $1962^{1}$, however, its implementation is problematic and consequently the method is still not in widespread use in acoustics.

One major impediment is that the solution is typically progressed from known initial conditions using a time marching scheme. This is an inherently iterative process and so has the potential for instability. Numerical error arising from discretisation, integration accuracy or machine precision may distort the stable behaviour of the physical scenario, causing the numerical model to diverge from the true solution. Modes of behaviour that 
are lightly damped are likely candidates for causing such instability since they are already close to the stability threshold, and therefore should be avoided where possible.

When the problem of sound scattering from a body is stated as a Boundary Integral Equation (BIE), the precursor to forming a BEM, the restriction that sound cannot travel through the body is lost and an air-filled cavity is effectively created inside the body's bounding surface. At certain frequencies this cavity may resonate, storing energy so the time-invariant frequency domain BEM has a non-unique solution. In the time domain problem, these resonances correspond to time-invariant oscillations, on the cusp of instability and likely candidates for corruption into divergence by numerical error. Such modes are not physically relevant, so their removal is acceptable and improves solver performance. One method that achieves this in the frequency domain is the Burton \& Miller formulation ${ }^{2}$, and it has been transferred to the time domain as the Combined Field Integral Equation (CFIE) ${ }^{3}$.

The creation of this complementary interior problem causes issues when the obstacle tapers to a thin fin. The sound in the virtual cavity becomes largely independent of the excitation sound, so the problem becomes ill-posed. A formulation has previously been published ${ }^{4}$ which addresses this issue for scattering by thin plates, but such an "all-thin" model is unsuitable for problems involving tapering obstacles, or obstacles with or in the presence of thin fins, due to the issues described in the previous paragraph. Therefore this paper aims to develop a formulation suitable for this more general scenario.

The BEM is a wave based method and its computational cost increases rapidly with frequency. Acceleration algorithms ${ }^{5,6,7}$ have been published to address this issue but, as 
these are derived from the time-marching solvers for which instability issues remain, the focus herein remains on modelling smaller problems in a non-accelerated fashion. In addition some interesting work has been done on alternative solvers ${ }^{8,9,10,11}$ that may be less sensitive to divergent poles than the current time-marching generation.

This paper is structured as follows: Section 3 introduces the boundary integral formulation of the scattering problem and the CFIE. Section 4 describes the existing formulation for thin plates, and describes how that can be adapted for a mixed body problem. The discretisation process and time-marching solver, and a technique for quantifying their stability are specified in sections 5 and 6 respectively. Verification results are shown and discussed in section 7 followed by the conclusions in section 8 Finally details of the numerical integration procedure are outlined in the appendix.

\section{Boundary Integral Equations}

This section introduces the Boundary Integral Equations which are needed to derive the new BEM in Section 4. Figure 1 depicts a scattering problem, comprising an obstacle submerged in a connected medium $\Omega_{+}$with equilibrium density $\rho_{0}$ which obeys the linear acoustic wave equation with speed of sound $c . S$ is a surface conformal to the obstacle and sufficiently close that the obstacle's surface properties may be ascribed to it, thus the obstacle resides in the interior domain $\Omega . . S_{\infty}$ is the extent of the medium. $\mathbf{x}$ and $\mathbf{y}$ are $3 \mathrm{D}$ Cartesian vectors defining the observation and radiation points respectively, $R=|\mathbf{x}-\mathbf{y}|$ is the distance between them and $\hat{\mathbf{n}}_{\mathbf{y}}$ is the surface normal unit vector at $\mathbf{y}$. 
Sound is represented by the velocity potential $\varphi$ which, while not a physical quantity, has the convenient property that both pressure $p$ and particle velocity $v$ may be derived from it:

$$
\begin{aligned}
& p(\mathbf{x}, t)=-\rho_{0} \dot{\varphi}(\mathbf{x}, t) \\
& \mathbf{v}(\mathbf{x}, t)=\nabla \varphi(\mathbf{x}, t)
\end{aligned}
$$

where $t$ is time and a dot above a quantity indicates temporal differentiation. An incident disturbance $\varphi_{i}(\mathbf{x}, t)$ exists in $\Omega_{+}$but does not reach the obstacle while $t \leq 0$. When $\varphi_{i}(\mathbf{x}, t)$ does reach the obstacle a wave $\varphi_{s}(\mathbf{x}, t)$ is scattered such that the total disturbance $\varphi_{t}(\mathbf{x}, t)=\varphi_{i}(\mathbf{x}, t)+\varphi_{s}(\mathbf{x}, t)$ matches the surface properties of the obstacle, thus this is an initial-boundary-value problem. Application of Greens Theorem ${ }^{12}$ allows the propagation of $\varphi_{s}(\mathbf{x}, t)$ in $\Omega_{+}$to be stated as the Kirchhoff Integral Equation (KIE) over its boundary $S \cup S_{\infty}$. In practice $S_{\infty}$ is chosen so distant that its contribution does not arrive within the modelling duration, so the integration domain may be reduced to $S$. The scattered velocity potential from a rigid obstacle is thus:

$$
\varphi_{s}(\mathbf{x}, t)=\iint_{S} \varphi_{t}(\mathbf{y}, t) * \hat{\mathbf{n}}_{\mathbf{y}} \cdot \nabla_{\mathbf{y}} g(R, t) d \mathbf{y}
$$

* denotes temporal convolution and $\mathrm{g}(R, t)$ is the time domain Greens function, which describes how sound travels from a point source to a point observer, given by: 


$$
g(R, t)=\frac{\delta(t-R / c)}{4 \pi R}
$$

where $\delta(t)$ is a dirac delta function.

Consideration of the obstacle's boundary condition at $\mathbf{x}$ allows solution for the total surface sound, from which scattered sound at any desired off-surface point may be evaluated.

\subsection{Pressure Operator}

The pressure operator $L_{p}$ is derived from the assertion that within the obstacle the total pressure must be zero, i.e. $p_{t}(\mathbf{x}, t)=0$ if $\mathbf{x} \in \Omega_{-}$. Recalling that the total sound is the sum of incident and scattered sound, and substituting Equations 1 and 3, yields:

$$
-\dot{\varphi}_{i}(\mathbf{x}, t)=\mathrm{L}_{\mathrm{p}}\left\{\varphi_{t}(\mathbf{x}, t)\right\}=\frac{\partial}{\partial t} \iint_{S} \varphi_{t}(\mathbf{y}, t) * \hat{\mathbf{n}}_{\mathbf{y}} \cdot \nabla_{\mathbf{y}} g(R, t) d \mathbf{y} \quad \text { if } \mathbf{x} \in S_{-}
$$

$\mathbf{x}$ must lie within $\Omega$. for the underlying assertion to hold but must also be on $S$ for total

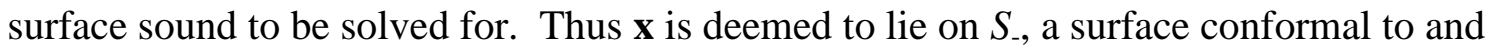
infinitesimally inside $S$. This choice weakens the above assertion and permits non-zero pressures in the cavity away from its boundary, hence $\mathrm{L}_{\mathrm{p}}$ supports soft cavity modes.

\subsection{Velocity Operator}

The velocity operator $L_{v}$ follows from the assertion that if the boundary is stationary then the surface normal component of particle velocity must be zero, i.e.

$\hat{\mathbf{n}}_{\mathbf{x}} \cdot \mathbf{v}_{t}(\mathbf{x}, t)=0$ if $\mathbf{x} \in S$. Recalling that total sound is the sum of incident and scattered sound, and substituting Equations 2 and 3, yields: 


$$
\hat{\mathbf{n}}_{\mathbf{x}} \cdot \nabla \varphi_{i}(\mathbf{x}, t)=\mathrm{L}_{\mathrm{v}}\left\{\varphi_{t}(\mathbf{x}, t)\right\}=-\hat{\mathbf{n}}_{\mathbf{x}} \cdot \nabla_{\mathbf{x}} \iint_{S} \varphi_{t}(\mathbf{y}, t) * \hat{\mathbf{n}}_{\mathbf{y}} \cdot \nabla_{\mathbf{y}} g(R, t) d \mathbf{y} \quad \text { if } \mathbf{x} \in S
$$

These statements only restrict the particle velocity at $S$ so waves in the cavity are permitted, including cavity resonances and pneumatic modes ${ }^{13}$.

\subsection{Combined Field Integral Equation Operator}

The CFIE operator $\mathrm{L}_{\mathrm{c}}$ is stated ${ }^{3}$ to be the time domain equivalent of the Burton and Miller formulation $^{2}$ commonly used in frequency domain acoustic BEMs. Its formulation differs slightly from the latter, in particular with regard to the range of values taken by the blend parameter $\alpha$, and matches more closely its namesake in the Electromagnetic BEM formulation ${ }^{14}$. It may be expressed in terms of a linear sum of $\mathrm{L}_{\mathrm{p}}$ and $\mathrm{L}_{\mathrm{v}}$ :

$$
\begin{aligned}
\mathrm{L}_{\mathrm{c}}\left\{\varphi_{t}(\mathbf{x}, t)\right\} & =(1-\alpha) \dot{\varphi}_{i}(\mathbf{x}, t)+\alpha c \hat{\mathbf{n}}_{\mathbf{x}} \cdot \nabla \varphi_{i}(\mathbf{x}, t) \\
& =-(1-\alpha) \mathrm{L}_{\mathrm{p}}\left\{\varphi_{t}(\mathbf{x}, t)\right\}+\alpha c \mathrm{~L}_{\mathrm{v}}\left\{\varphi_{t}(\mathbf{x}, t)\right\} \quad \text { if } \mathbf{x} \in S_{-}
\end{aligned}
$$

It is equivalent to the boundary condition $(1-\alpha) p_{t}(\mathbf{x}, t)=\alpha \rho_{0} c \hat{\mathbf{n}}_{\mathbf{x}} \cdot \mathbf{v}_{t}(\mathbf{x}, t)$ if $\mathbf{x} \in S_{-}$, which when $\alpha=1 / 2$ is satisfied by any plane wave propagating in the direction of $\hat{\mathbf{n}}_{\mathbf{x}}$ out of the cavity. More generally, it has been shown that when $0<\alpha<1$ energy flows out of the cavity and it cannot support resonant modes ${ }^{15}$. Consequently, the application of $\mathrm{L}_{\mathrm{c}}$ has been shown to grant superior stability to $\mathrm{L}_{\mathrm{p}}$ and $\mathrm{L}_{\mathrm{v}}$ for a variety of test geometries. Therefore it is desirable to derive the BEM algorithm from the CFIE for all compatible scattering obstacles. 


\section{Obstacles with thin appendages}

So far discussion has focussed on obstacles that have significant interior volume.

However, there are various devices used in acoustics which have thin parts, for example an orchestral canopy or the Schroeder diffuser that will be modelled later in this paper.

All thin objects occurring in the real world have some finite thickness, so accordingly attempts have been made to use the closed surface BEM to model these with two surfaces, each conformal to a body-air interface. This is a reasonable model of reality, however, when the BEM is applied problems can occur. In particular, the complementary interior problem is now an extremely thin cavity, so areas of the cavity walls opposite one another interact very strongly, dominating over their interactions with other parts of the body and making the problem ill-posed. This phenomenon is known as Thin-Shape Breakdown (TSB) and has been found to cause frequency-independent illconditioning in the solution stage of frequency domain BEM models ${ }^{16}$. The TSB also affects the time domain BEM, where it is most likely to manifest as solver instability.

TSB can be avoided by taking the limit as the thickness approaches zero and approximating the two body-air interfaces by a single surface. This approach has the added benefit of reducing the number of surface elements hence improves computational efficiency. Figure 2 depicts a thin body with surfaces $S_{1}$ and $S_{2}$, and the scattering from it may be written: 


$$
\varphi_{s}(\mathbf{x}, t)=\iint_{S_{1}} \varphi_{t}\left(\mathbf{y}_{1}, t\right) * \hat{\mathbf{n}}_{\mathbf{y}_{1}} \cdot \nabla_{\mathbf{y}_{1}} g(R, t) d \mathbf{y}_{1}+\iint_{S_{2}} \varphi_{t}\left(\mathbf{y}_{2}, t\right) * \hat{\mathbf{n}}_{\mathbf{y}_{2}} \cdot \nabla_{\mathbf{y}_{2}} g(R, t) d \mathbf{y}_{2}
$$

As the thickness approaches zero $S_{1}$ approaches $S_{2}$, so $\mathbf{y}_{1}$ and $\mathbf{y}_{2}$ coalesce. The normal vectors are opposed but the gradient of the Greens function is continuous, hence the sound radiated by the surface is a function of the jump in velocity potential (or pressure) across the surface instead of the absolute values on each side. This may be written as a single integral:

$$
\varphi_{S}(\mathbf{x}, t)=\iint_{S} \tilde{\varphi}_{t}(\mathbf{y}, t) * \hat{\mathbf{n}}_{\mathbf{y}} \cdot \nabla_{\mathbf{y}} g(R, t) d \mathbf{y}
$$

where the surface subscripts are dropped as they both refer to the same surface, and $\tilde{\varphi}_{t}(\mathbf{y}, t)=\varphi_{t}\left(\mathbf{y}_{1}, t\right)-\varphi_{t}\left(\mathbf{y}_{2}, t\right)$ is jump velocity potential. $S$ is now physically interpreted as a thin rigid shell that resists pressure, an air-air interface.

Since $\varphi_{t}\left(\mathbf{y}_{1}, t\right)$ and $\varphi_{t}\left(\mathbf{y}_{2}, t\right)$ are both unknown, no boundary condition of the form $\varphi_{t}\left(\mathbf{y}_{2}, t\right)=0$ may be used. However, boundary conditions involving the surface normal component of velocity are still valid, so the rigid surface boundary condition $\hat{\mathbf{n}}_{\mathbf{x}} \cdot \mathbf{v}_{t}(\mathbf{x}, t)=0$ if $\mathbf{x} \in S$ may be applied; this is simply $\mathrm{L}_{\mathrm{v}}$ applied to jump velocity potential.

$$
\hat{\mathbf{n}}_{\mathbf{x}} \cdot \nabla \varphi_{i}(\mathbf{x}, t)=-\hat{\mathbf{n}}_{\mathbf{x}} \cdot \nabla \varphi_{s}(\mathbf{x}, t)=\mathrm{L}_{\mathrm{v}}\left\{\tilde{\varphi}_{t}(\mathbf{x}, t)\right\} \quad \text { if } \mathbf{x} \in S
$$

The above operator permits solution for a jump velocity potential field on $S$ that satisfies the rigid obstacle boundary condition for a given incident sound wave. Total velocity 
potential at any point off $S$ may be calculated using Equation 9, including $\mathbf{y}_{1}$ and $\mathbf{y}_{2}$ if the limit is taken as $\mathbf{x}$ approaches $S$ from the appropriate side.

This approach of modelling scattering by thin plates was implemented in the time domain by Kawai and Terai ${ }^{4}$. However there remains the question of what is best when it is desired to model a plate near a solid body, or a solid body with a protruding fin. It transpires that Equation 10 may be used for the entirety of such a mixed problem, as for the air-body interfaces of the non-thin sections $\varphi_{t}\left(\mathbf{y}_{2}, t\right)=0$ hence $\tilde{\varphi}_{t}(\mathbf{y}, t)=\varphi_{t}\left(\mathbf{y}_{1}, t\right)$; this will be referred to as an "all-thin" model. However, Ergin et al ${ }^{3}$ highlighted that $\mathrm{L}_{\mathrm{v}}$ supports cavity resonances and so is unreliable in this application; instead they promote use of the CFIE on closed bodies.

$\mathrm{Wu}^{17}$ addressed the same concerns in the frequency domain and showed that nonuniqueness occurs if any closed bodies exist in a mixed body problem. He implemented a BEM that uses different solution strategies on thick and thin surface parts; thin parts were modelled with the surface normal derivative of the KIE, as above, closed parts were modelled with either the Burton and Miller method ${ }^{2}$ or the CHIEF method ${ }^{18}$, both of which inhibit cavity resonances and the associated non-uniqueness issue.

Wu was careful to distinguish between closed and open surfaces. In contrast, the comments on jump potential above suggest that these surface parts need not be distinguished in this way; they merely have differing boundary conditions. Derivation is further simplified because $\mathrm{L}_{c}$ reduces to $\mathrm{L}_{v}$ when $\alpha=1$; this is not the case for the Burton and Miller definition in which the KIE and its surface normal derivative have a fixed weight of one and a variable weight respectively. 
Consequentially the mixed surfaces algorithm proposed here is to use the CFIE with the replacement of $\varphi^{t}\left(\mathbf{r}^{\prime}, t\right)$ by $\tilde{\varphi}^{t}\left(\mathbf{r}^{\prime}, t\right)$ and the constraint that $\alpha=1$ on thin surface sections. This requires the meshing scheme to choose which sections of the obstacle will be modelled by closed surfaces and which by open surfaces at time of discretisation, so problems could still be encountered for tapering bodies for which it is unclear how and when is best to switch between representations. In this application the numerical integration implementation described in Appendix A excels as it regularises all integrands, so singularities in non-self element interactions are correctly handled. It must also be ensured that thin appendages do not bisect body elements, and that meshing of a tapering section is symmetrical, since contravening either could make the boundary fields unsmooth at the collocation point, the consequences of which have not been considered. The numerical solution strategy will now be described.

\section{Marching-On-in-Time}

The surface quantities must be discretised in order for a solution to the boundary conditions on $S$ to be found numerically. The scheme described here follows that published in reference 3, except that the numerical integration uses an improved implementation which is more accurate.

The surface $S$ is partitioned into $N_{s}$ flat elements denoted $S_{n}$, all small with respect to the anticipated spatial variation of the sound field, and time is discretised into $N_{t}$ regular time-steps with duration $\Delta_{t}$. Discretisation of the incoming wave is achieved by approximating it by a weighted summation of basis functions: 


$$
\varphi_{t}(\mathbf{x}, t)=\sum_{n=1}^{N_{s}} \sum_{i=1}^{N_{t}} w_{n, i} \mathrm{f}_{n}(\mathbf{x}) \mathrm{T}_{i}(t)
$$

where $w_{n, i}$ are the discretisation weights, $\mathrm{f}_{n}(\mathbf{x})$ are the spatial basis functions, which are unity if $\mathbf{x} \in S_{n}$ or zero otherwise, and $\mathrm{T}_{i}(t)=\mathrm{T}\left(t-i \Delta_{t}\right)$ are the temporal basis functions, the latter being regularly delayed copies of the mother basis function $\mathrm{T}(t)$. Herein $\mathrm{T}(t)$ is chosen to be the piecewise polynomial used in reference 3 .

This discretisation scheme is substituted into Equation 7 and the summations and weights are brought outside $\mathrm{L}_{\mathrm{c}}$. Collocation is performed in space and time to form a matrix equation; evaluation at $\mathbf{x}_{m}$ (the centre of element $S_{m}$ ) and $t_{j}=j \Delta_{t}$ contributes a row to:

$$
\mathbf{Z}_{0} \mathbf{w}_{j}=\mathbf{e}_{j}-\sum_{l=1}^{\infty} \mathbf{Z}_{l} \mathbf{w}_{j-l}
$$

where $l=j-i$ is the retardation index and the weights $\mathbf{w}_{i ; n}=w_{n, i}$. As the collocation points are in the centre of elements the surface and velocity potential field are guaranteed to by smooth nearby, and the boundary condition consistent. This simplifies evaluation of the interaction matrices, which are defined as:

$$
\mathbf{Z}_{l ; m, n}=\mathrm{L}_{\mathrm{c}}\left\{\mathrm{f}_{n}(\mathbf{x}) \mathrm{T}_{j-l}\left(t_{j}\right)\right\} \quad \mathbf{x}=\mathbf{x}_{m-}
$$

where the CFIE blend coefficient $\alpha$ is chosen to be $1 / 2$ or 1 for thick or thin elements respectively. These are evaluated efficiently and accurately by regularisation to contour integrals and adaptive numerical integration; details are included in Appendix A. These will be zero for $l$ greater than some constant due to finite $c$. The excitation vectors are evaluated as: 


$$
\mathbf{e}_{j ; m}=(1-\alpha) \dot{\varphi}_{i}\left(\mathbf{x}_{m}, t_{j}\right)+\alpha c \hat{\mathbf{n}}_{\mathbf{x}} \cdot \nabla \varphi_{i}\left(\mathbf{x}_{m}, t_{j}\right)
$$

This algorithm is commonly referred to as the Marching On in Time (MOT) or 'Retarded Potential' algorithm, and intuitively possesses an iterative structure with sound travelling from element to element with a finite speed. It may more generally be considered to be a matrix solver between excitation coefficients and discretisation weights, which exploits a pattern in the interaction matrices due to the regular temporal basis functions.

Discretisation accuracy may be quantified spatially and temporally by considering the maximum frequency $\omega_{\max }$ present in the incident wave. The maximum phase variation over an element with largest dimension $\Delta_{x}$ in a time-step is $\omega_{\max }\left(\Delta_{t}+\Delta_{x} / \mathrm{c}\right)$. The logical assumption that spatial and temporal discretisation error should be of similar magnitudes

suggests the choice $c \Delta_{t} \approx \Delta_{x}$, as favoured by Bluck and Walker ${ }^{20}$. This leads to non-zero off-diagonals in the matrix $\mathbf{Z}_{0}$, necessitating a matrix solution at each time-step. $\mathbf{Z}_{0}$ will in practice be very sparse and an iterative matrix solver seeded with the previous timestep's weights provides an efficient implementation.

\section{BEM stability analysis}

Stability is a crucial issue for the time domain BEM so the vast majority of publications touch upon stability issues, and many propose conditions (often heuristic) that if met guarantee stability of the corresponding algorithm. Similar instabilities affect all time marching BEM models regardless of the field of application, implying that this behaviour is fundamental to the solution method, and these instabilities commonly take the form of an exponentially increasing oscillation that alternates in sign at each time-step ${ }^{19}$. 
The dominant mathematical analysis of this phenomenon is the Singularity Expansion Method (SEM) $)^{20,21,22}$ which expands the continuous time system response into a sum of damped exponentials with poles $s_{n}$ and corresponding spatial modes $\boldsymbol{\Phi}_{n}$ :

$$
\left.\boldsymbol{\Phi}^{t}(t)=\sum_{n} \boldsymbol{\Phi}_{n} \mid \operatorname{Re}\left\{e^{s_{n} t}\right\} * \boldsymbol{\Phi}_{n} \cdot \boldsymbol{\Phi}^{i}(t)\right]
$$

where $\boldsymbol{\Phi}^{i}(t)$ represents system excitation. This can be written in discrete time as:

$$
\boldsymbol{\Phi}_{j}^{t}=\sum_{n} \boldsymbol{\Phi}_{n}\left[\operatorname{Re}\left\{\lambda_{n}^{j}\right\}_{j}^{*} \boldsymbol{\Phi}_{n} \cdot \boldsymbol{\Phi}_{j}^{i}\right]
$$

where the discrete time pole $\lambda_{n}=e^{s_{n} \Delta_{t}}$, hence $\operatorname{Re}\left\{s_{n}\right\}=\ln \left|\lambda_{n}\right| / \Delta_{t}$ and $\operatorname{Im}\left\{s_{n}\right\}=\angle \lambda_{n} / \Delta_{t}$. The initial conditions prohibit such solutions, so they must be initiated by excitation or numerical error. Multiple divergent modes may exist simultaneously but, due to their exponential growth, the one with the largest magnitude pole will ultimately dominate.

For a stable solution $\forall \operatorname{Re}\left\{s_{n}\right\} \leq 0$ or equivalently $\forall\left|\lambda_{n}\right| \leq 1$ must be satisfied. Poles of the underlying physical scenario are stable as are those of the BIE, although the latter may possess extra poles on the cusp of stability if it supports cavity resonances. Any pole may be perturbed by discretisation and numerical $\operatorname{error}^{23}$, so borderline stable poles are likely candidates for divergence. High frequency poles have poor spatial and temporal resolution so experience the worst error, explaining why the dominant pole often has a phase of $\pm \pi$ or equivalently a frequency of $\left(2 \Delta_{t}\right)^{-1}$.

Once excitation has ceased an iteration of the MOT equation may be represented by multiplication of a state history vector $\mathbf{h}_{j}$ by the state-transition matrix $\mathbf{M} . \mathbf{M}$ is non- 
normal and does not possess a full set of linearly independent eigenvectors. However, there is evidence that it's largest magnitude eigenvalues often correspond to the discrete time poles $\lambda_{n}$ of recognisable modes ${ }^{20,21,22}$. , For a typical mesh, $\mathbf{M}$ is very large (though sparse) so hardware storage and precision often limit the size of problem for which $\mathbf{M}$ may be constructed and any eigenvalues resolved.

In the following section numerical results will be presented. Where possible the largest magnitude state-transition eigenvalues will be calculated and displayed as a measure of stability.

\section{Results}

The modified algorithm for mixed bodies will be verified by comparison with frequency domain BEM implementations which have previously been shown to accurately match experimental data ${ }^{24}$, and by considering the transient response and largest magnitude SEM poles. Two scattering problems are considered, both of which involve obstacles possessing thin fins, the first being a simple cube with a square fin attached, and the second being a Schroeder Diffuser.

One spatial mesh is used for each surface, and the time domain BEM is tested over a wide range of time-step durations defined by their relationship to spatial resolution, denoted implicitness $c \Delta_{t} / \Delta_{x}$. This is done because time-step duration has been associated with stability in many publications; evidently different values affect whether poles are perturbed into divergence. 
In order to quantify accuracy, a harmonic point source illuminates the surface for sufficient duration that the system reaches steady state and any instability has the opportunity to appear. The time domain surface velocity potential is discrete Fourier transformed and the percentage error versus the frequency domain BEM is quantified at the frequency of excitation; the details of this process are found in Appendix B. This error was analysed as a contour plot versus time-step implicitness and temporal resolution $\beta=2 \pi / \omega \Delta_{t}$ and the trends observed are described in what follows. The ranges of timestep implicitness and temporal resolution tested were $-1 \leq \log _{10}\left(c \Delta_{t} / \Delta_{x}\right) \leq 1$ and $5 \leq \beta \leq$ 20 respectively, equating to a frequency range from $12 \mathrm{~Hz}$ to nearly $5 \mathrm{kHz}$. Using single frequency excitation is clearly an uninspiring application of the time domain BEM but is being done purely to achieve rigorous verification.

\subsection{Cube with a thin fin}

This mesh is conceived as a simple test case for the CFIE mixed surfaces model. It comprises a $0.7 \mathrm{~m}$ cube with a $0.7 \mathrm{~m}$ fin attached. $\Delta_{x}=0.1 \mathrm{~m}$ so the cube has 294 thick elements and the fin 49 thin elements. It is an extremely regular mesh with all elements having an equal aspect ratio. Care is taken to ensure that the fin's line of attachment does no bisect any body elements. It is depicted in Figure 3 (thin elements are coloured blue online).

First the cube section of the mesh was modelled alone to ensure that the BEM implementation of the CFIE was performing correctly. The model was universally stable and error compared with the frequency domain BEMs was generally better than 5\% when $c \Delta_{t} / \Delta_{x}>1 / 2$ and $\beta>10$, and, as good as $1 \%$ when $\beta$ approached 20 . 
Having verified the cube as a closed body problem, investigations progressed to including the fin, so as to verify the implementation for a mixed body problem. Stability and accuracy trends remained unchanged, confirming correctness of the proposed scheme for mixed surfaces.

The decay of the impulse response of the system is expected to be dominated by the least damped pole, hence this characterises the BEM model's stability and realism. Figure 4 shows the transient behaviour of the cube plus fin problem mixed model for $c \Delta_{t}=\Delta_{x}$. The data series plotted are total pressure for two receivers located interior to and exterior but close to the body. In order for the decay of the system to be visible and to excite poles over a wide frequency range, the excitation was required to be extremely short and pulse like in nature. A true Dirac delta pulse cannot be used in the current implementation because it is discontinuous; instead a Hanning plane wave was chosen as it is smooth with compact support:

$$
\begin{aligned}
\varphi_{i}(\mathbf{x}, t) & =A\left[1-\cos \left(\frac{2 \pi}{d}\left(t-t_{0}-\frac{\hat{\mathbf{i}} \cdot \mathbf{x}}{c}\right)\right)\right] \\
& \times\left[\mathrm{H}\left(t-t_{0}-\frac{\hat{\mathbf{i}} \cdot \mathbf{x}}{c}\right)-\mathrm{H}\left(t-t_{0}-\frac{\hat{\mathbf{i}} \cdot \mathbf{x}}{c}-d\right)\right]
\end{aligned}
$$

where $H(\ldots)$ is the Heavyside function, and the parameters were chosen to take the values amplitude $A=10^{-3}$, duration $d=0.02 \mathrm{~s}$, start time $t_{0}=0.1 \mathrm{~s}$, and propagation vector $\hat{\mathbf{i}}=\{0,0,-1\}$. The incident wave is clearly visible in Figure 4 (note Equation 1) dominating the response at the receiver external to the cube. 
In order to observe the decay of the scattered field a $\mathrm{dB}$ scale is required, hence this is used in Figure 5, which is otherwise identical to Figure 4. Pressure inside the scattering body is $35 \mathrm{~dB}$ lower than outside; this quantifies the completeness of cancellation between incident and scattered waves and would be expected to be greater for more slowly varying waves. Following this pressure at both receivers decays at approximately $3500 \mathrm{~dB} / \mathrm{s}$, indicating that all poles are extremely well damped and consistent with the modelled scenario being a convex body devoid of features associated with acoustic resonance.

Figure 6 is a graphical representation of how time-step duration affects the SEM poles of the discrete system. The state transfer matrix $\mathbf{M}$ was constructed ${ }^{20}$ and the largest of its eigenvalues found numerically; these are estimates of the discrete time poles $\lambda_{n}$ and their magnitudes are plotted against the time-step implicitness $c \Delta_{t} / \Delta_{x}$. The continuous time poles $s_{n}$ should ideally be invariant of $\Delta_{t}$, but the magnitudes of their discrete counterparts vary with $\Delta_{t}^{-1}$; where these can be identified they have been indicated and their loci plotted. The pole at $s=0$ is typical of boundary conditions involving the derivatives of $\varphi$ and corresponds to a silent mode where velocity potential is unchanged between timesteps hence $p=0$. All poles of the cube plus fin problem mixed model are stable.

Figure 7 shows results from the same scenario as Figure 5 but modelled with an all-thin boundary condition; this response represents what would be calculated by previously published time-domain BEMs. The behaviour is initially very similar, with pressure inside the scattering body again $35 \mathrm{~dB}$ lower than outside, but then the decay of the external pressure is much slower at only $263 \mathrm{~dB} / \mathrm{s}$, implying that the system response is less well damped so possibly less realistic. The interior pressure decays briefly but stalls 
at a constant value approximately $95 \mathrm{~dB}$ lower than peak incident pressure, which could be a pneumatic $(0 \mathrm{~Hz})$ mode as investigated by $\operatorname{Parot}^{13}$, but is certainly not a behaviour representative of the physical problem modelled. In addition, high frequency oscillations in the surface quantities were observed for the shortest (most explicit) time-step durations modelled which is clearly erroneous.

Fewer eigenvalues have been resolved in Figure 8 than Figure 6, and those shown display less identifiable trends suggesting they are more corrupted, plus have larger magnitudes. The largest magnitude pole (excluding $s \approx 0$ ) at $\log _{10}\left(c \Delta_{t} / \Delta_{x}\right)=0$ is indicated and has a value $s \approx-28+1400 j$. This closely matches the decay rate observed in Figure 7 , providing strong evidence that it is this pole that dominates the scattered pressure for short duration plane wave excitation. Another pole indicated at $\log _{10}\left(c \Delta_{t} / \Delta_{x}\right)=0.4$ oscillates divergently at the Nyquist frequency; this did not dominate in the durations modelled as verification cases, but with an unfortunate choice of excitation it would.

These mechanisms observed for the transient response also compromise the harmonic response, giving an unacceptable error average of around $83 \%$ compared to the frequency domain BEM. Thus the superiority of the mixed body scheme in terms of accuracy and stability is confirmed for a simple scattering problem.

\subsection{Schroeder Diffuser}

Room acoustic diffusers are passive devices which attempt to scatter sound uniformly over a range of frequencies. They can be used to treat critical listening environments to improve speech intelligibility and to make music sound better ${ }^{25}$. Their scattering may be measured under anechoic conditions ${ }^{26}$, but this is a time consuming and expensive 
process. An alternative is to predict this dispersion using a numerical model, and the BEM is well suited to this task ${ }^{27}$. The speed and low cost of this approach aids prototyping of new designs, and even allows automated optimisation of treatments to be performed $^{28}$.

The development of phase grating diffusers can be traced back to the pioneering work of Schroeder ${ }^{29,30}$. They comprise a series of wells of differing depths according to a number theoretic sequence, separated by thin fins, and sound waves entering each well emerge following the time taken for them to travel to the bottom of the well, reflect and travel back to the mouth. These delays are optimally decorrelated so the cumulative scattered sound is widely dispersed. Because the wells store sound energy and then reradiate it, the scattered sound is diffused in both space and time, hence their transient response has recently begun to attract research interest ${ }^{31,32}$.

A Schroeder diffuser is an ideal example of an obstacle to be modelled using the new mixed body algorithm as it comprises a closed body and thin fins and possesses no tapering sections, and Cox and $\mathrm{Lam}^{27}$ have shown that such a frequency domain BEM model is accurate in this application. In addition, they compared results with a model where the behaviour of each well is approximated by a surface impedance at its mouth; this algorithm has also recently been successfully implemented in the time domain ${ }^{33}$.

In this paper, a one-dimensional diffuser based on the Quadratic Residue Sequence [2 41 $\left.\begin{array}{lll}0 & 1 & 4\end{array}\right]$ will be considered; these are designed to diffuse in one plane only and take the form of an extruded cross section. The design wavelength was chosen to be approximately $1.4 \mathrm{~m}$, the well width $0.25 \mathrm{~m}$, and the height $1.0 \mathrm{~m} . \Delta_{x}=0.1 \mathrm{~m}$ meaning the 
mesh comprises 726 thick elements and 174 thin elements; it is depicted in Figure 9 where the thin elements are shown partially transparent (and blue online).

First the QRD was modelled without its fins; this was therefore an obstacle with a series of steps on the front face. Use of the CFIE was stable for all but the shortest two timestep durations modelled, and the error trend versus the frequency domain BEM changed little from the from the cube mesh (generally better than $5 \%$ when $c \Delta_{t} / \Delta_{x}>1 / 2$ and $\beta>$ 12), except that it was interrupted by a localised error increase of a few per-cent close to the diffuser design frequency. This artefact also appeared in a comparison between the two frequency domain BEM implementations, and a state-transition eigenvalue can be resolved with similar natural frequency; the cause and significance of this behaviour being at the design frequency is an ongoing research question. In contrast, use of an allthin model resulted in instability for 7 of the 21 time-step durations modelled, and an average error of approximately $300 \%$ for the stable results, again reinforcing the superiority of the CFIE for closed obstacles.

Subsequently the fins were reinstated and the model repeated. The obstacle now contains a wealth of exterior convex parts and parallel surfaces, whose presence suggests a disposition toward resonance. Indeed each well may be considered to be a $1 / 4$ wave resonator, albeit damped by energy leaving through its mouth. Energy trapped in these is not suppressed by the CFIE as they are physically relevant external features of the device; their response is part of the desired solution. It will be seen if discretisation errors push these lightly damped poles into instability or if accuracy is maintained. 
For the QRD mixed model, underlying error trends compared to the frequency domain BEM were harder to identify than previous models due to the presence of instability and a localised error maxima at $136 \mathrm{~Hz}$, a frequency with no obvious geometrical significance. In general accuracy was poorer than has previously been observed, typically between $3 \%$ and $30 \%$ where $c \Delta_{t}>2 \Delta_{x}$ and $\beta>10$. However this performance should be placed in context against the all-thin model which is unstable for the majority of time-step durations, with the remaining showing poorly damped behaviour and error rarely lower than $100 \%$; clearly this is a very challenging modelling scenario.

Figure 10 shows the resolvable state-transition eigenvales of the QRD mixed model, being estimates of the discrete-time SEM poles. At two time-step durations divergent poles can be clearly seen at the top of the figure both with frequency $\left(2 \Delta_{t}\right)^{-1}$. In addition divergent poles are also present for four more time-step durations (all explicit $c \Delta_{t}<<\Delta_{x}$ ) but hardware limitations mean they are not quantified in this figure. A few loci are identified for the corresponding continuous-time poles, but these are mostly nonoscillatory and none appear to correspond to characteristic physical resonances such as the $1 / 4$ wave resonant frequencies of the wells, or modes along or across the wells. The exception is the pole at $s \approx-69+860 j$ (red online) which matches the frequency of localised error increase mentioned above. It only appears for a short range of time-step durations since on the left of the figure there is corruption by instability, and on the right low pass filtering by the low sample rate. As was emphasised for the QRD block, the error maxima compared to the frequency domain results is accompanied by oscillatory eigenvalues, implying the presence of lightly damped oscillatory poles. This is unsurprising since the time harmonic problem effectively considers the equilibrium 
pressure at which incident and scattered energy are equal, and the damping or $Q$ of a resonance greatly affects its peak value. It appears that the largest magnitude statetransition eigenvalues dominate the overall accuracy of transient BEM results, so the accuracy with which they match the SEM poles of the physical problem may be critical.

The transient response is shown in Figure 11, where time-step duration is chosen such that $\log _{10}\left(c \Delta_{t} / \Delta_{x}\right)=0.1$ because the model is unstable when $c \Delta_{t}=\Delta_{x}$. Despite the error versus the frequency domain BEM at the surface being larger than for the cube plus fin mesh, this scattered response is more similar to the good behaviour in Figure 4 than the poorly-damped ill-behaviour in Figure 7; the scattered field appears at least subjectively plausible. Cancellation achieved inside the QRD is similar with pressure 33dB lower than outside, and the pressure at both receivers decays rapidly at approximately 2170 $\mathrm{dB} / \mathrm{s}$. The interior and exterior pressures appear to both stall at constant values $214 \mathrm{~dB}$ and $225 \mathrm{~dB}$ lower than incident respectively. However closer examination reveals that these are in fact exponentially growing instabilities, albeit growing extremely slowly at $2.3 \times 10^{-5} \mathrm{~dB} / \mathrm{s}$, which matches the magnitude of the largest state-transition eigenvalue being $1+1.44 \times 10^{-9}$; evidently the pole at $s=0$ has been perturbed to a slightly positive value. This behaviour is non-physical but the numerical errors involved are smaller than the numerical integration precision so blame probably lies there. However, despite insignificance within this modelling duration, presence of this artefact in an otherwise well behaved model does highlight the shortcomings of the current solution strategy.

Lastly, Figure 12 shows the same transient response as predicted by the all-thin model; this represents what would be calculated by previously published time-domain BEMs. Results are much poorer with cancellation inside the body reduced to $22 \mathrm{~dB}$, both 
receivers showing a slow decay of $92.1 \mathrm{~dB} / \mathrm{s}$ while oscillating at $127 \mathrm{~Hz}$, and the interior pressure approaching a constant value $67 \mathrm{~dB}$ lower than incident pressure. This model has been shown to be inaccurate compared to frequency-domain BEMs at the principle frequency of excitation, and clearly its transient response is unrealistic too.

There is an interest among diffuser designers as to the transient behaviour of their devices. This data is both scattering angle and time dependent, so presentation as a polar impulse response such as Figure 13 could be appropriate. Scattered velocity potential magnitude is plotted in $\mathrm{dB}$, versus angle normal to the diffuser and time, for a $5 \mathrm{~m}$ radius arc of receivers above the diffuser. Excitation was by a point source located $10 \mathrm{~m}$ distant normal to the diffuser. Display on Cartesian axes was felt superior to display on polar axes (where time would be the radius) because the latter could be misinterpreted as an instantaneous snapshot of sound in space, which it is not.

In order to aid temporal resolution a very short (explicit) time-step duration has been chosen $\left(\log _{10}\left(c \Delta_{t} / \Delta_{x}\right)=-0.5\right)$. Accuracy here is poor $(>30 \%)$ and further research and modelling is required to improve it. However like Figure 11, the transient response appears subjectively plausible, so this figure is included as an indicator of how future verified results may appear. Indeed it bears great qualitative resemblance to measured data published by Farina ${ }^{31}$. Verification against such measured transient data is a future objective for development of this algorithm.

\section{Conclusions}

Regular BEM implementations cannot model objects with thin fins as the proximity of the surfaces at each solid / air interface causes singular behaviour in the underlying 
integral equations, a phenomenon known as Thin Shape Breakdown. This has been tackled by adopting an open surfaces model where a single surface models the fin as a rigid air / air interface, an approach previously used with the time domain BEM so not itself new. However, it has also been shown that such an all-thin model of a closed surface permits cavity resonances so is often unstable. In light of this it was proposed herein that the open surfaces model be applied solely to the thin surface sections (the fins), the CFIE be applied to the remaining closed sections, and that an improvement in stability will result relative to an all-thin model. This mixed body approach is analogous to an approach used for the frequency domain BEM but is novel for the time domain algorithm.

The algorithm was verified on the simple problem of a cube with an attached fin; accuracy was good and stability universal. A single period of a real world device, a Quadratic Residue Diffuser, was then modelled. The approach was successful, achieving vastly superior stability and accuracy compared to the all-thin model, but some instability was still witnessed. It is suggested that this occurs because the wells are lightly damped resonators, so the corresponding poles are not suppressed by the CFIE as they are external to the body and physically relevant, and that these are easily corrupted into instability. Obviously the real device does not radiate exponentially increasing pressures so the behaviour of the time domain BEM is erroneous.

It is felt that deriving a heuristic criterion that might ensure stability for this particular modelling problem is not a research avenue that has wide scope. Instead, the results suggest that further research is required into achieving stability of the time domain BEM when modelling lightly damped acoustic resonators, such as wells and parallel plates. It 
has been inferred herein that if a lightly damped pole is not accurately calculated it causes substantial error in the time harmonic case close to its natural frequency. Similarly lightly damped poles dominate the transient response, so perhaps a measure of their perturbation would be an appropriate indicator of transient error, albeit one with substantial computational cost.

\section{Acknowledgements}

This project was been funded by the UK Engineering and Physical Sciences Research Council (EPSRC) under grant number GR/P01144/01.

\section{Appendix A}

Accurate evaluation of the interaction coefficients defined in Equation 13 is fundamental to the accuracy and stability of the algorithm. The temporal basis function has discontinuous derivatives which cause discontinuities and delta functions in the surface integrands, making them unsuitable for solution by Gaussian integration. In addition, the integrand is singular so element self-interaction need often be considered as a special case.

The implementation herein exploits the flat elements and piecewise-constant spatial basis functions to permit regularisation of all integrands by coordinate transformation ${ }^{4,34}$ such that the collocation point is no longer a special case. The radial component of integration is performed analytically, leaving the remaining numerical integration a one-dimensional contour integral. This allows an adaptive numerical integration scheme to be used, specifically Simpson integration with Romberg extrapolation. An absolute termination 
criterion was used, meaning that larger more significant interaction coefficients were evaluated with higher precision than smaller less significant ones. This process is arbitrarily accurate, has better computational cost scaling than two-dimensional integration, and allows the same integration routine to be used for all element pairs as effort is automatically concentrated where necessary.

In order to clarify the conversion of the surface integral over $S_{n}$ into nested integrals two new coordinate systems will be used; one is a cartesian system $(v, w, z)$ and one a cylindrical polar system $(r, \theta, z)$, both shown in Figure 14. The origin and positive $z$ direction are the same in both coordinate systems. The origin is defined as the projection of the collocation point $\mathbf{x}$ into the plane of $S_{n}$ and the positive $z$ direction is specified by $\hat{\mathbf{n}}_{\mathbf{y}}$. The positive $v$ direction is defined as the projection of $\hat{\mathbf{n}}_{\mathbf{x}}$ into the plane of $S_{n}$, such that $\hat{\mathbf{w}} \cdot \hat{\mathbf{n}}_{\mathbf{x}}=0$. The positive theta direction is defined such that $v=r \cos (\theta)$ and $w=r \sin (\theta)$ in the conventional way. The variable $z$ refers to the $z$ coordinate of the collocation point $\mathbf{x}$ and any reference to $v, w, r$ or $\theta$ implies the integration point $\mathbf{y}$.

For this scheme of flat elements with piecewise-constant spatial basis functions the sound scattered according to the discretised KIE may be expressed as follows, an angular integral over the edge of $S_{n}$ and a contribution from the origin:

$$
\varphi_{s}(\mathbf{x}, t)=\sum_{n=1}^{N_{s}} \sum_{i=1}^{N_{t}} w_{n, i}\left[\frac{\theta_{\text {origin }}}{4 \pi} \frac{z}{|z|} \mathrm{T}_{i}\left(t-\frac{|z|}{c}\right)-z \oint_{S_{n}} \frac{\mathrm{T}_{i}(t-R / c)}{4 \pi R} d \theta\right]
$$

$\theta_{\text {origin }}$ is the angle the edges of $S_{n}$ make around the origin. This is zero if the origin is outside $S_{n}$ and $2 \pi$ if $S_{n}$ contains the origin. If the origin lies on an edge $\theta_{\text {origin }}$ will equal 
the enclosed angle; intersection of one edge implies $\theta_{\text {origin }}=\pi$, intersection of a corner implies $\theta_{\text {origin }}$ will equal the acute angle between the adjoining edges.

The interaction coefficients for the CFIE are numerically evaluated by:

$$
\begin{aligned}
& \mathbf{Z}_{l ; m, n}=\frac{1}{4 \pi} \sum_{\text {edges } 0} \int^{1}\left[\begin{array}{l}
{\left[\begin{array}{l}
\left(1-\alpha-\alpha \hat{\mathbf{n}}_{\mathbf{x}} \cdot \hat{\mathbf{n}}_{\mathbf{y}} \frac{z}{R}\right) \frac{d \theta}{d \mu} \\
+\alpha \hat{\mathbf{n}}_{\mathbf{x}} \cdot \hat{\mathbf{v}} \frac{1}{R} \frac{d w}{d \mu}
\end{array}\right] z \frac{z}{R} \dot{\mathrm{T}}_{j-l}\left(t_{j}-\frac{R}{c}\right)} \\
+\alpha \frac{c}{R}\left[\begin{array}{l}
\hat{\mathbf{n}}_{\mathbf{x}} \cdot \hat{\mathbf{n}}_{\mathbf{y}}\left(1-\frac{z^{2}}{R^{2}}\right) \frac{d \theta}{d \mu} \\
+\hat{\mathbf{n}}_{\mathbf{x}} \cdot \hat{\mathbf{v}} \frac{z}{R^{2}} \frac{d w}{d \mu}
\end{array}\right] \mathrm{T}_{j-l}\left(t_{j}-\frac{R}{c}\right)
\end{array}\right] d \mu \\
& -\frac{\theta_{\text {origin }}}{4 \pi}\left[1-\alpha-\alpha \hat{\mathbf{n}}_{\mathbf{x}} \cdot \hat{\mathbf{n}}_{\mathbf{y}} \frac{z}{|z|}\right] \frac{z}{|z|} \dot{\mathrm{T}}_{j-l}\left(t_{j}-\frac{|z|}{c}\right)
\end{aligned}
$$

Numerical integration is with respect to $\mu$; a dimensionless edge position coefficient varying from zero at the start vertex to one at the end vertex. For an edge $\mathbf{e}$ the partial differentials between this and the geometric integration variables are found as follows where $r_{\perp}$ is the minimum (perpendicular) distance from the origin to the line of edge $\mathbf{e}$ : 


$$
\begin{aligned}
& \frac{d \theta}{d \mu}=\frac{|\mathbf{e}| r_{\perp}}{r^{2}} \operatorname{sign}(\hat{\mathbf{e}} \cdot \hat{\boldsymbol{\theta}}) \\
& \frac{d w}{d \mu}=\hat{\mathbf{w}} \cdot \mathbf{e}
\end{aligned}
$$

\section{Appendix B}

Two frequency domain BEM implementations were used for verification purposes; a closed body version equivalent to $\mathrm{L}_{\mathrm{p}}$ and an open body version equivalent to $\mathrm{L}_{\mathrm{v}}$. Both use piecewise constant interpolation and have previously been verified against experimental results ${ }^{24}$. The closed body BEM can only be used on the closed sections of meshes, but its inclusion was necessary as the open body BEM showed evidence of encountering non-unique solutions at certain frequencies.

For the harmonic problems the source is located in the far-field, 100m distant normal to the obstacle. This excites the system at a frequency such that the number of time-steps per excitation period $\beta=2 \pi / \omega \Delta_{t}$ assumes a range of predetermined integer values. For each combination the error $e$ between the time and frequency domain BEMs is calculated from the normalised mean complex difference between the respective source-tocollocation-point transfer functions at the excitation frequency: 


$$
e(\omega)=\frac{\sum_{m=1}^{N_{s}}\left|\mathrm{H}_{T D}\left(\mathbf{x}_{m}, \omega\right)-\mathrm{H}_{F D}\left(\mathbf{x}_{m}, \omega\right)\right|}{\sum_{m=1}^{N_{s}}\left|\mathrm{H}_{F D}\left(\mathbf{x}_{m}, \omega\right)\right|}
$$

In the frequency domain the transfer function $\mathrm{H}_{F D}$ is simply the total pressure divided by the source monopole pressure:

$$
\mathrm{H}_{F D}(\mathbf{x}, \omega)=\frac{P_{t}(\mathbf{x}, \omega)}{P_{\text {source }}}
$$

In the time domain $\mathrm{H}_{T D}$ is found by division of the discrete Fourier transform (DFT) of the total velocity potential by the DFT of the source monopole potential:

$$
\mathrm{H}_{T D}(\mathbf{x}, \omega)=\frac{F\left\{\varphi_{t}(\mathbf{x}, t)\right\}(\omega)}{F\left\{\varphi_{\text {source }}(t)\right\}(\omega)}
$$

The first $50 \beta$ iterations are omitted from the DFT to allow the time domain solution to reach steady state. The next $100 \beta$ iterations are chosen for DFT; this length maintains periodicity and eliminates windowing error. This error ratio was analysed as a contour plot versus time-step implicitness and temporal resolution $\beta$ and the trends observed are described herein. 
ACTA ACUSTICA UNITED WITH ACUSTICA Vol. 95 (2009) 678 - 689 DOI 10.3813/AAA.918196

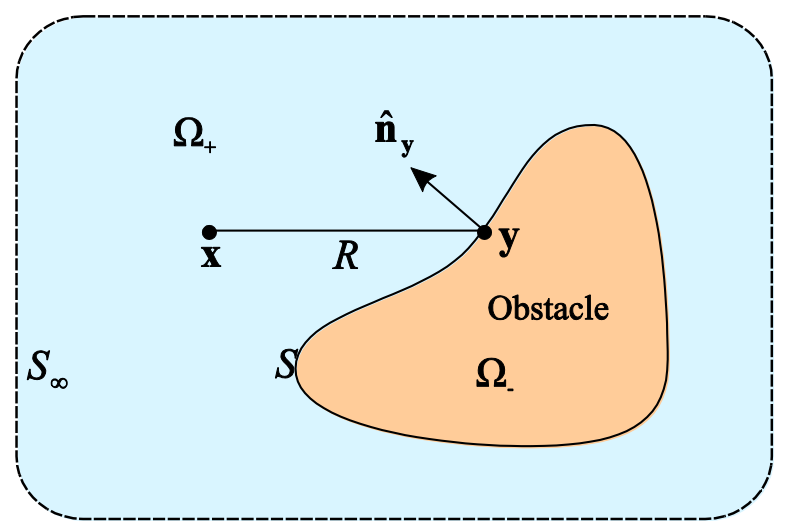

Figure 1 (Captions at end of document)

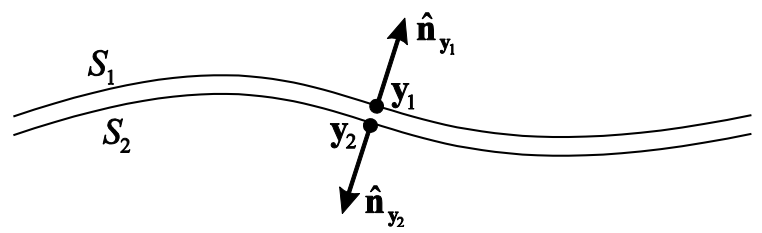

Figure 2 


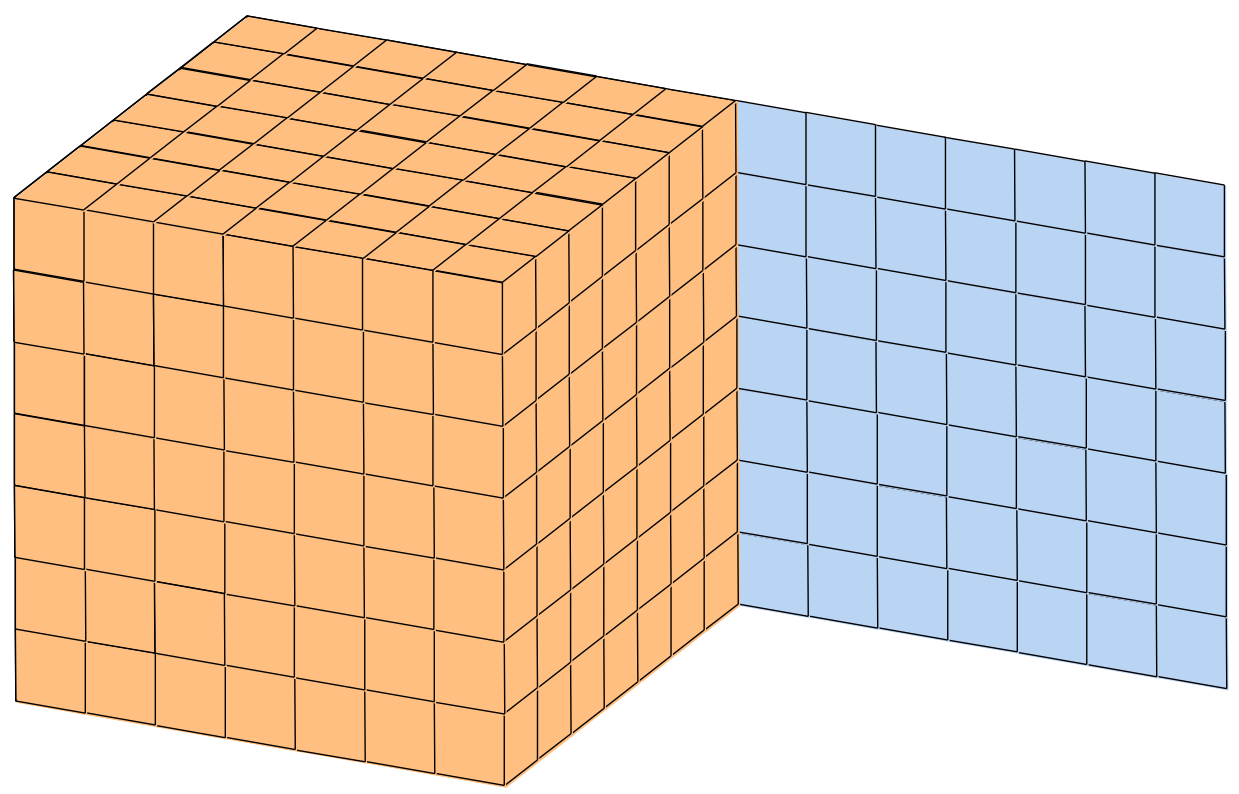

Figure 3 


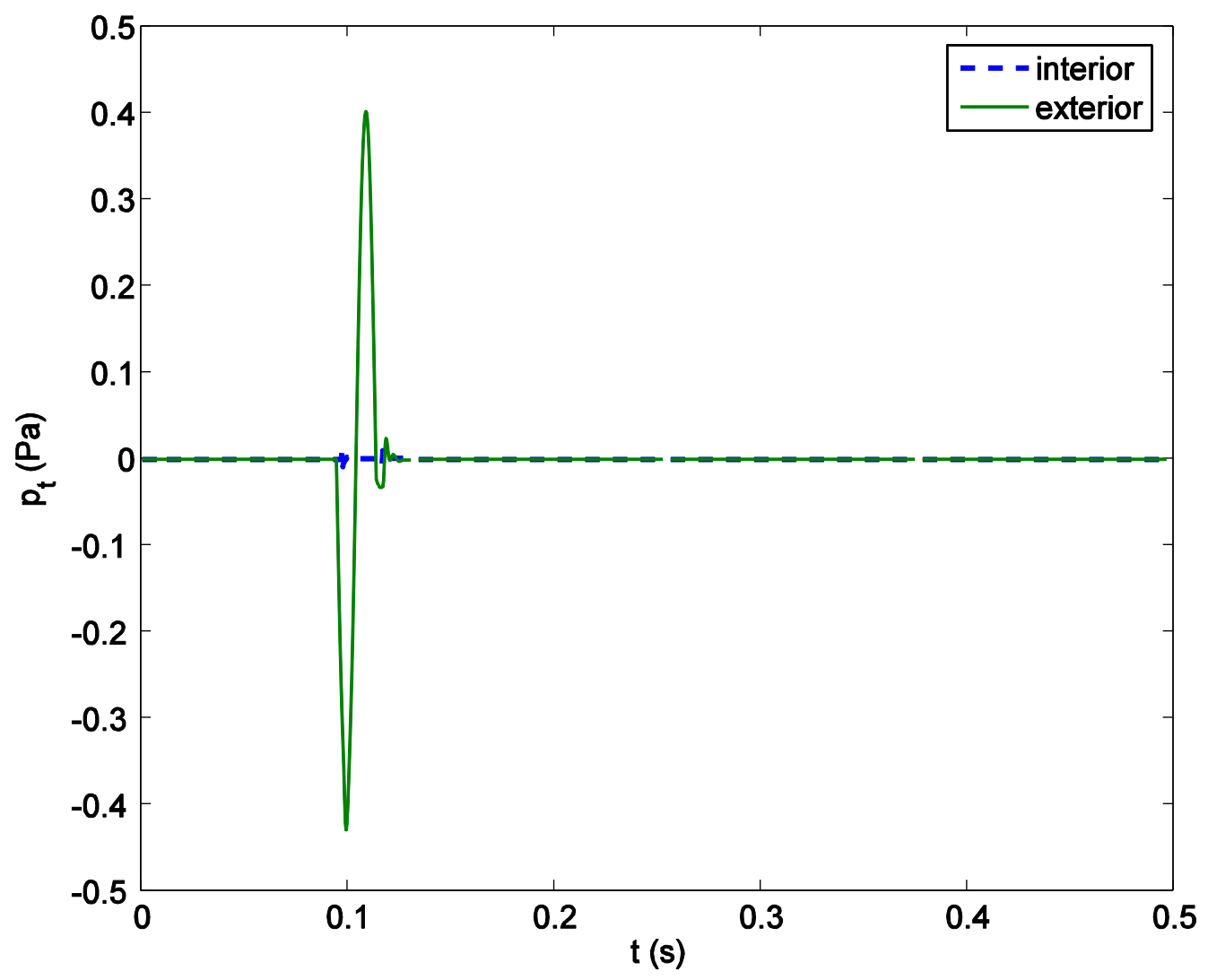

Figure 4 


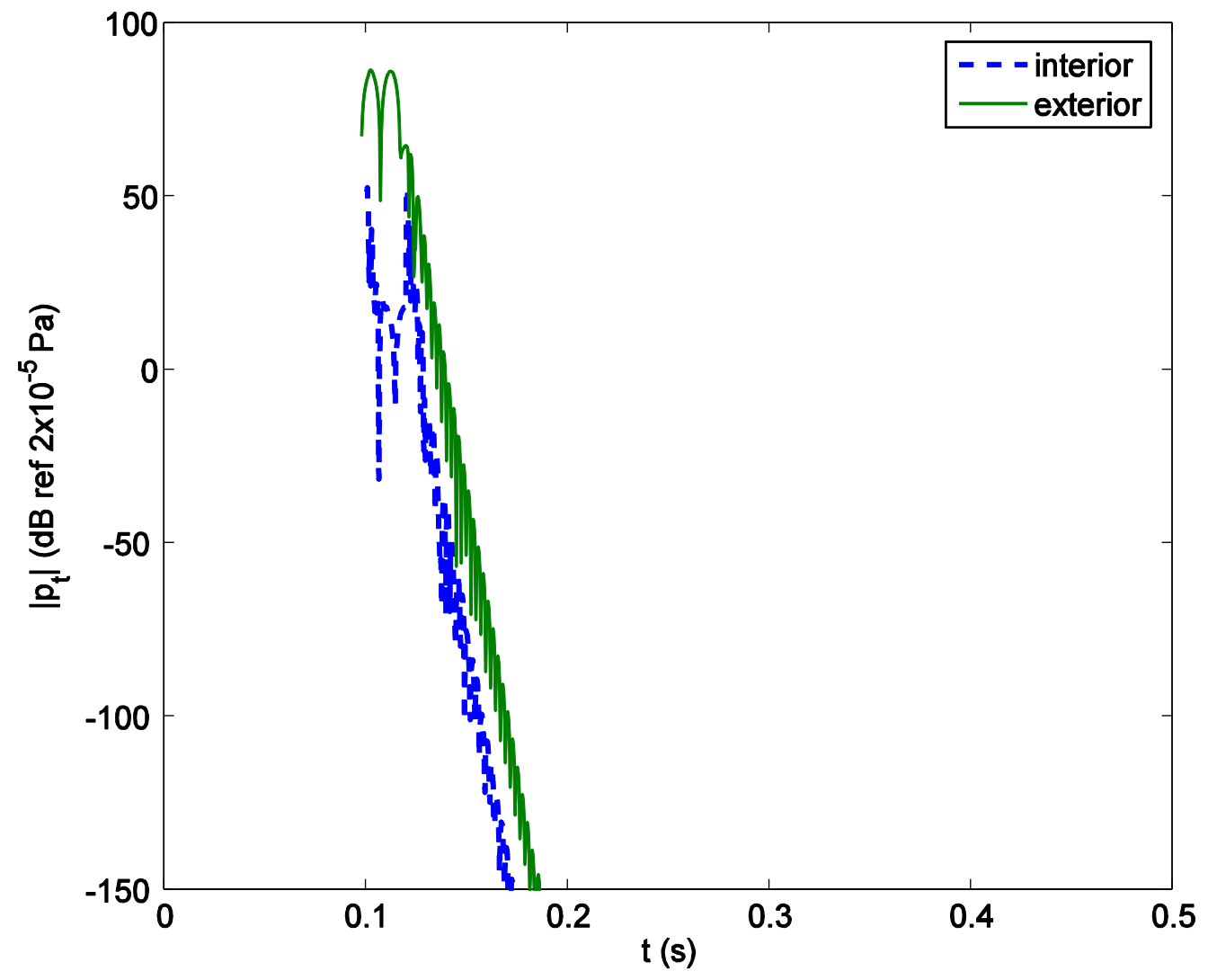

Figure 5 
ACTA ACUSTICA UNITED WITH ACUSTICA Vol. 95 (2009) 678 - 689 DOI 10.3813/AAA.918196

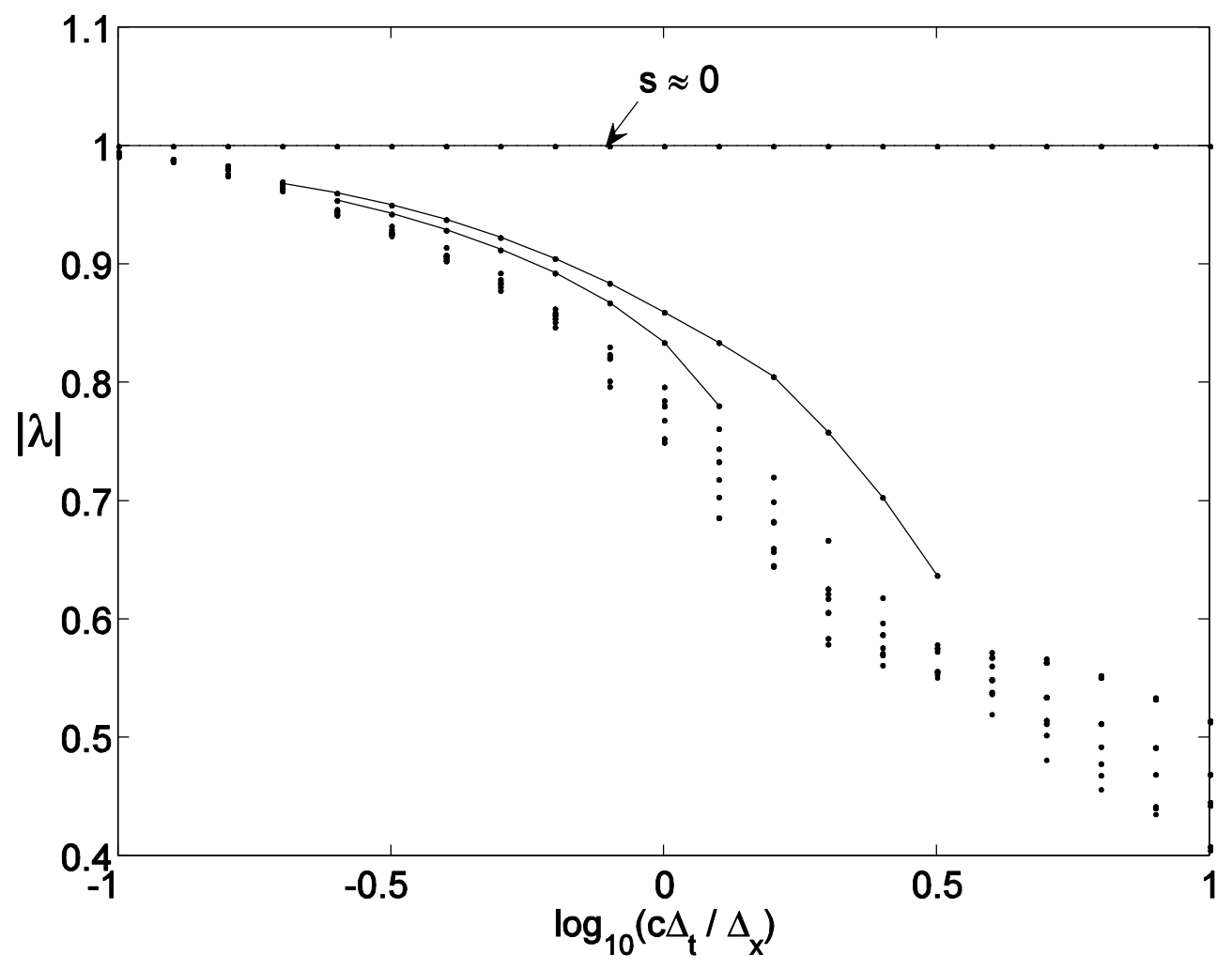

Figure 6 


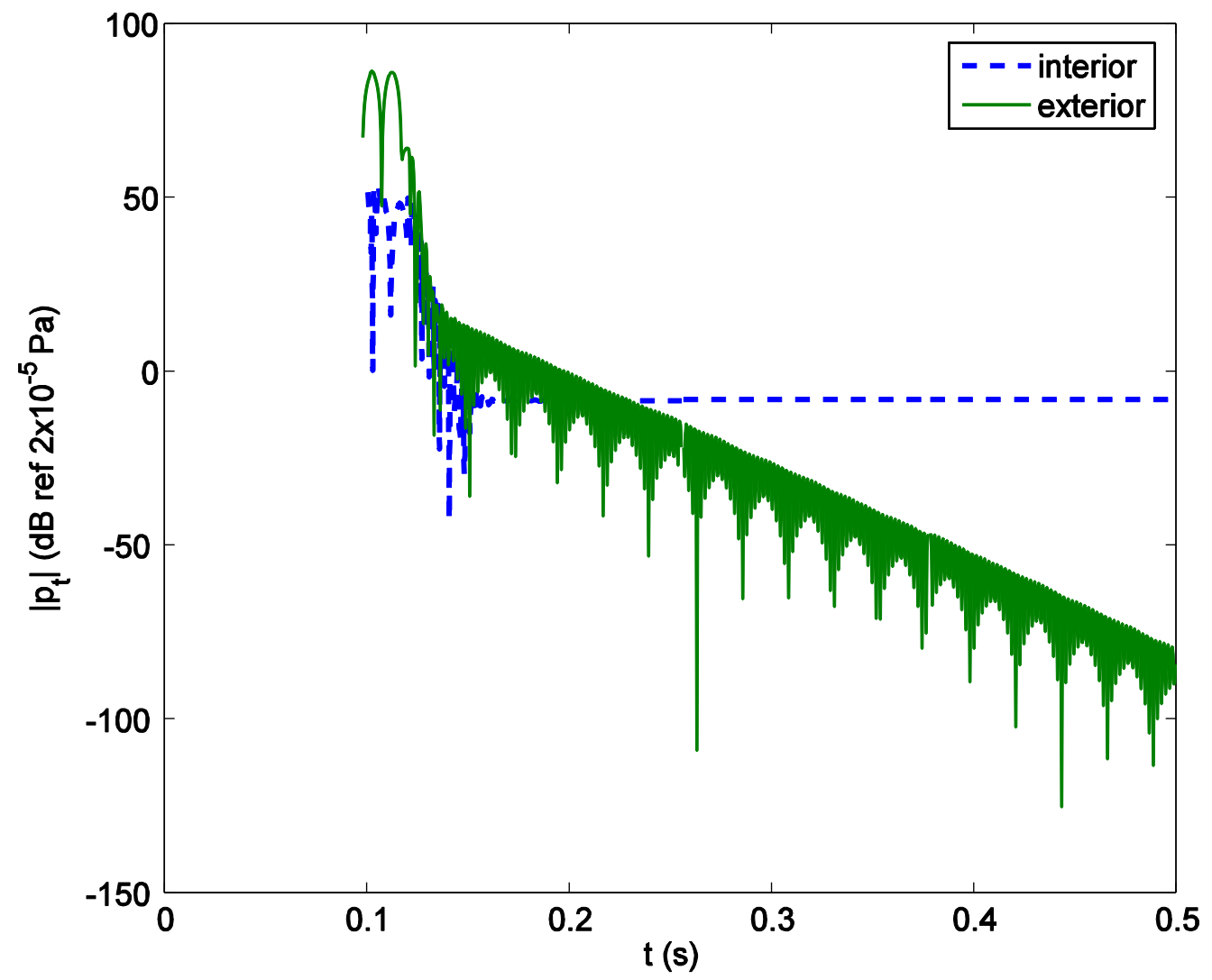

Figure 7 


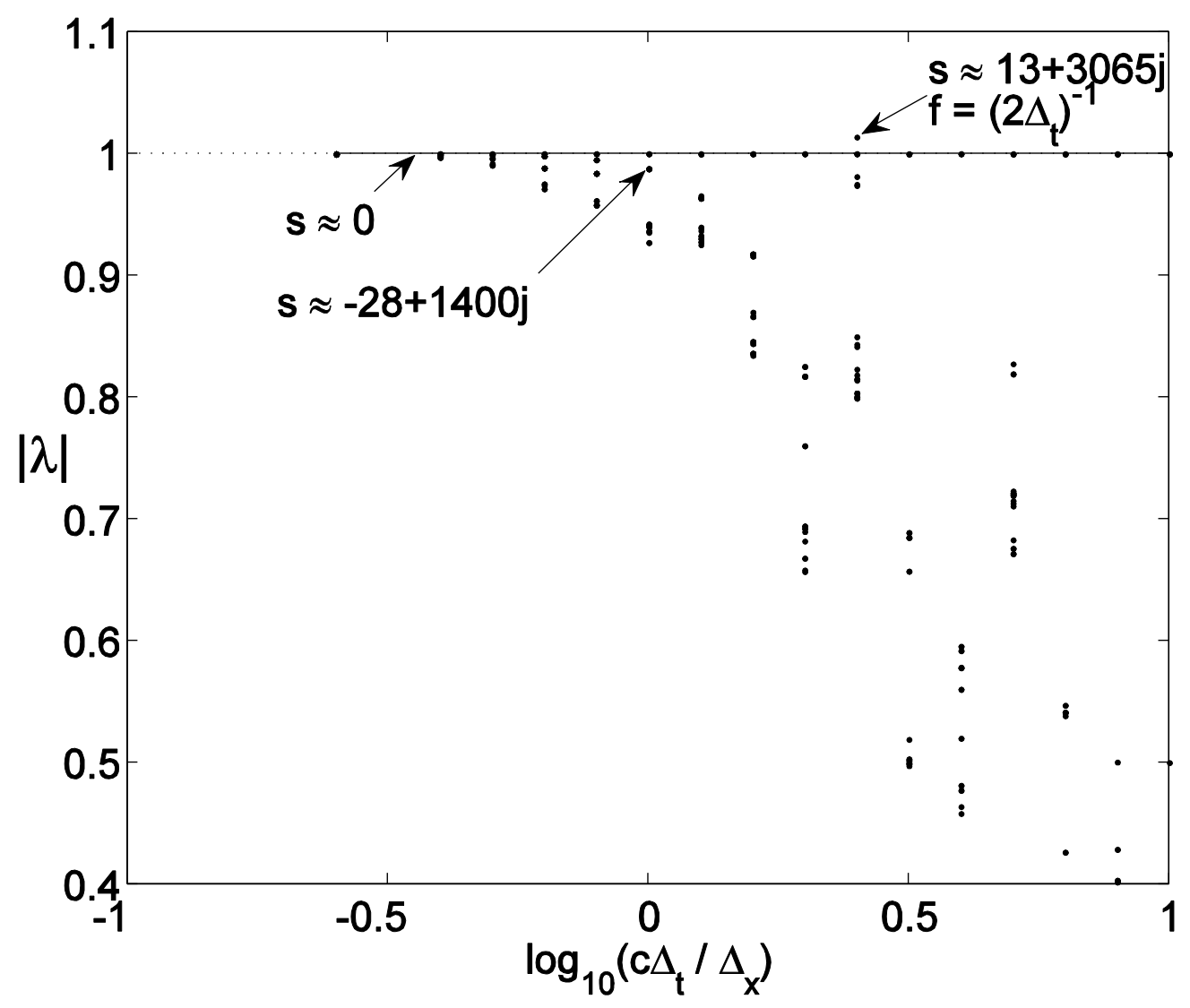

Figure 8 


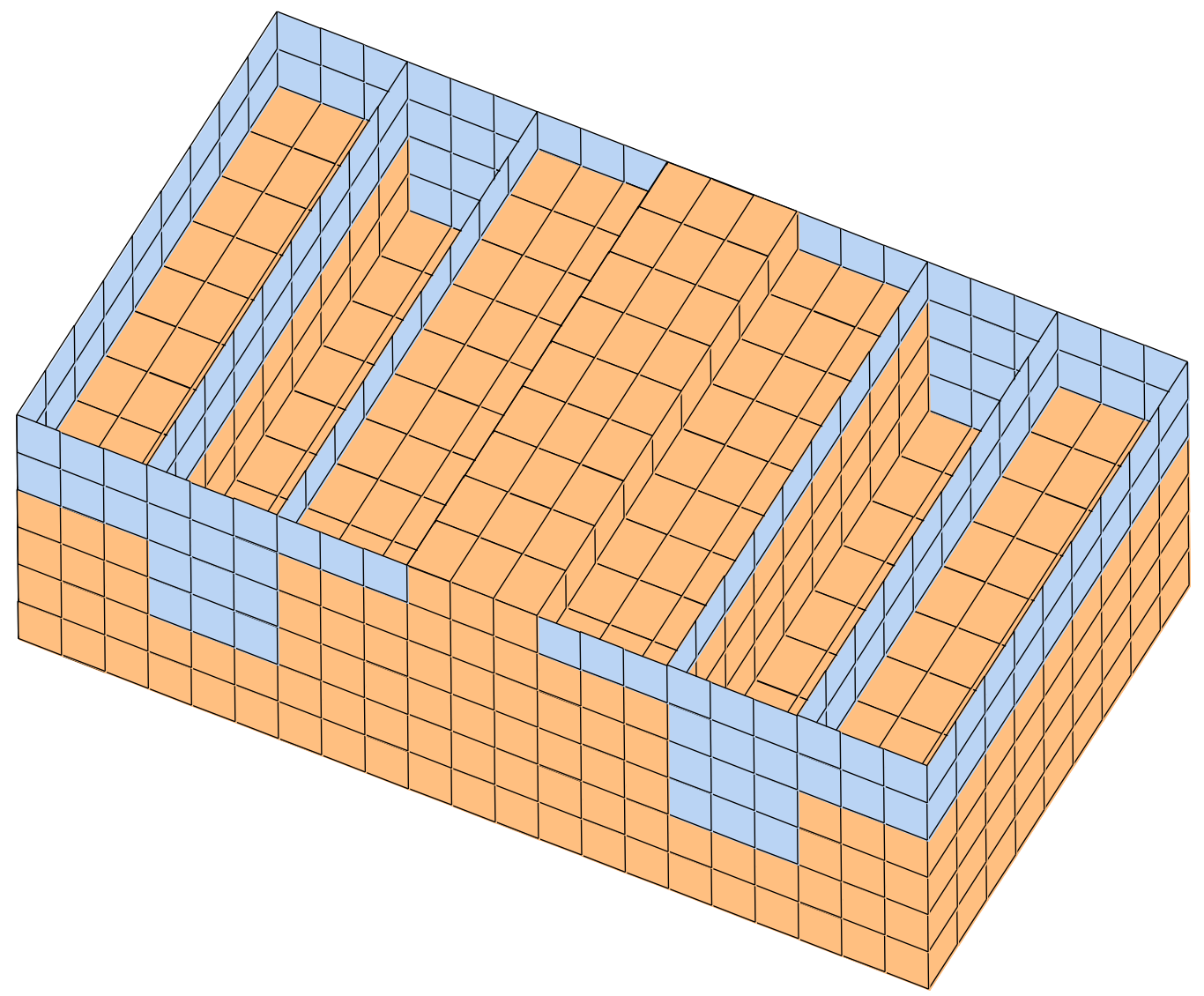

Figure 9 


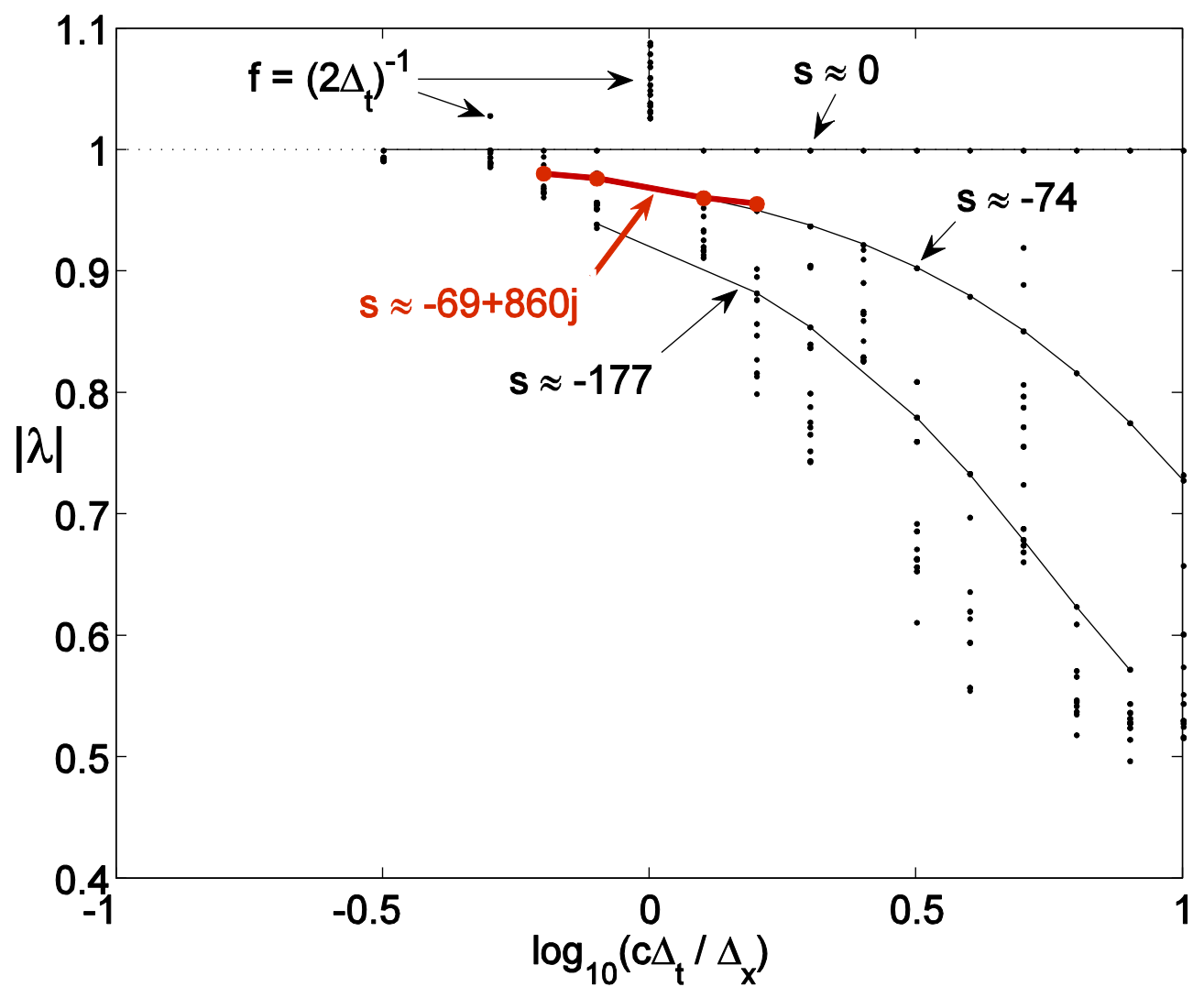

Figure 10 


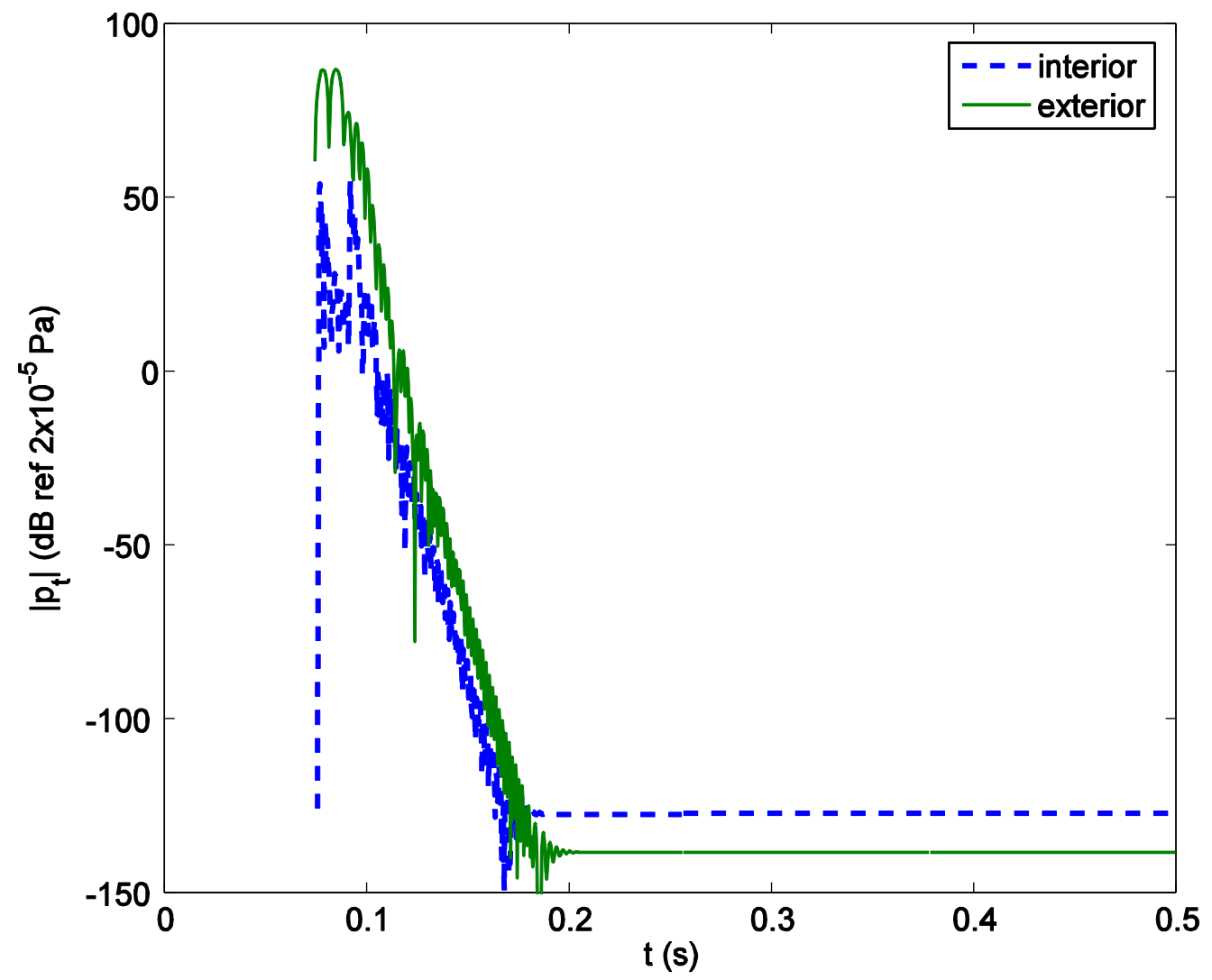

Figure 11 


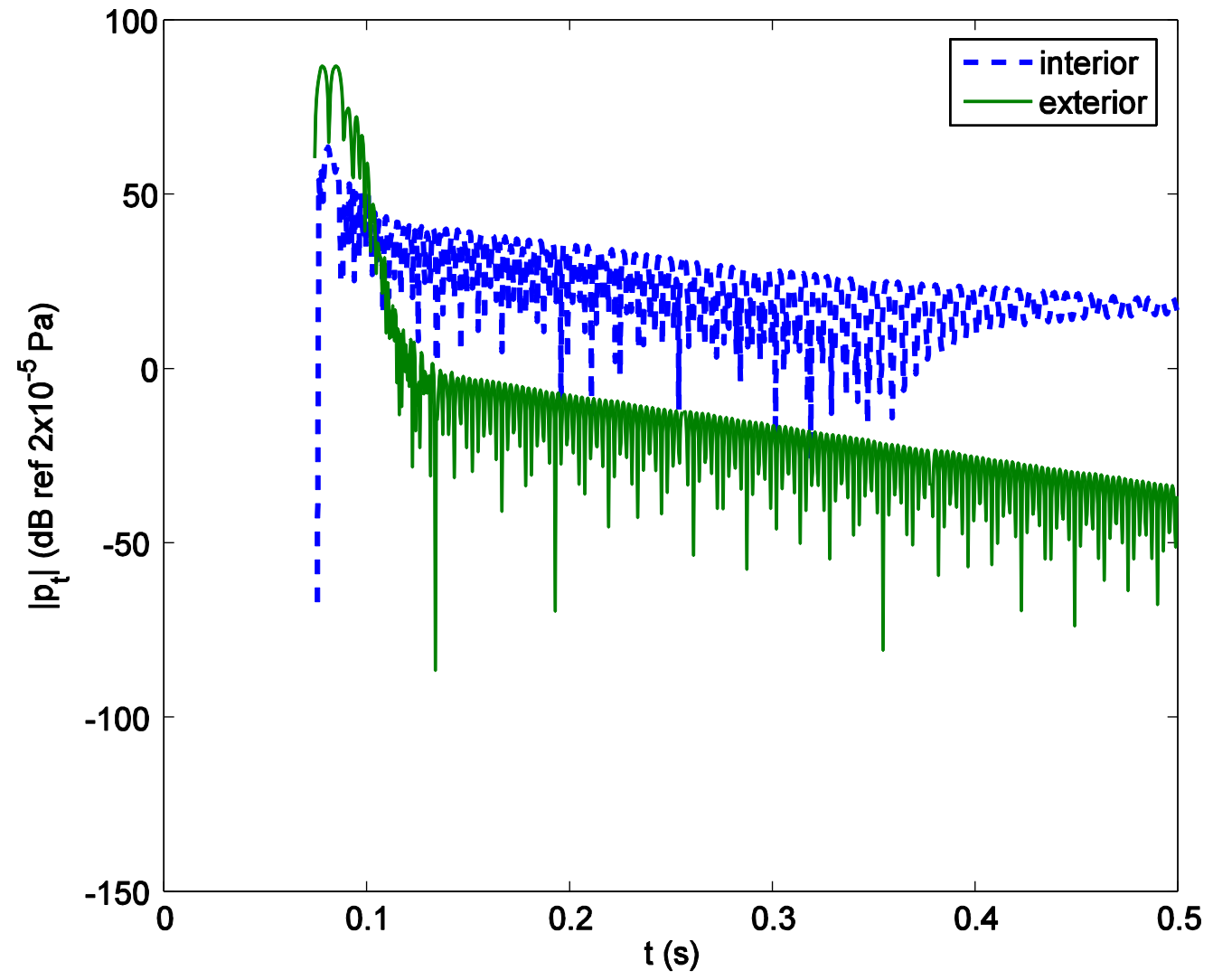

Figure 12 
ACTA ACUSTICA UNITED WITH ACUSTICA Vol. 95 (2009) 678 - 689 DOI 10.3813/AAA.918196

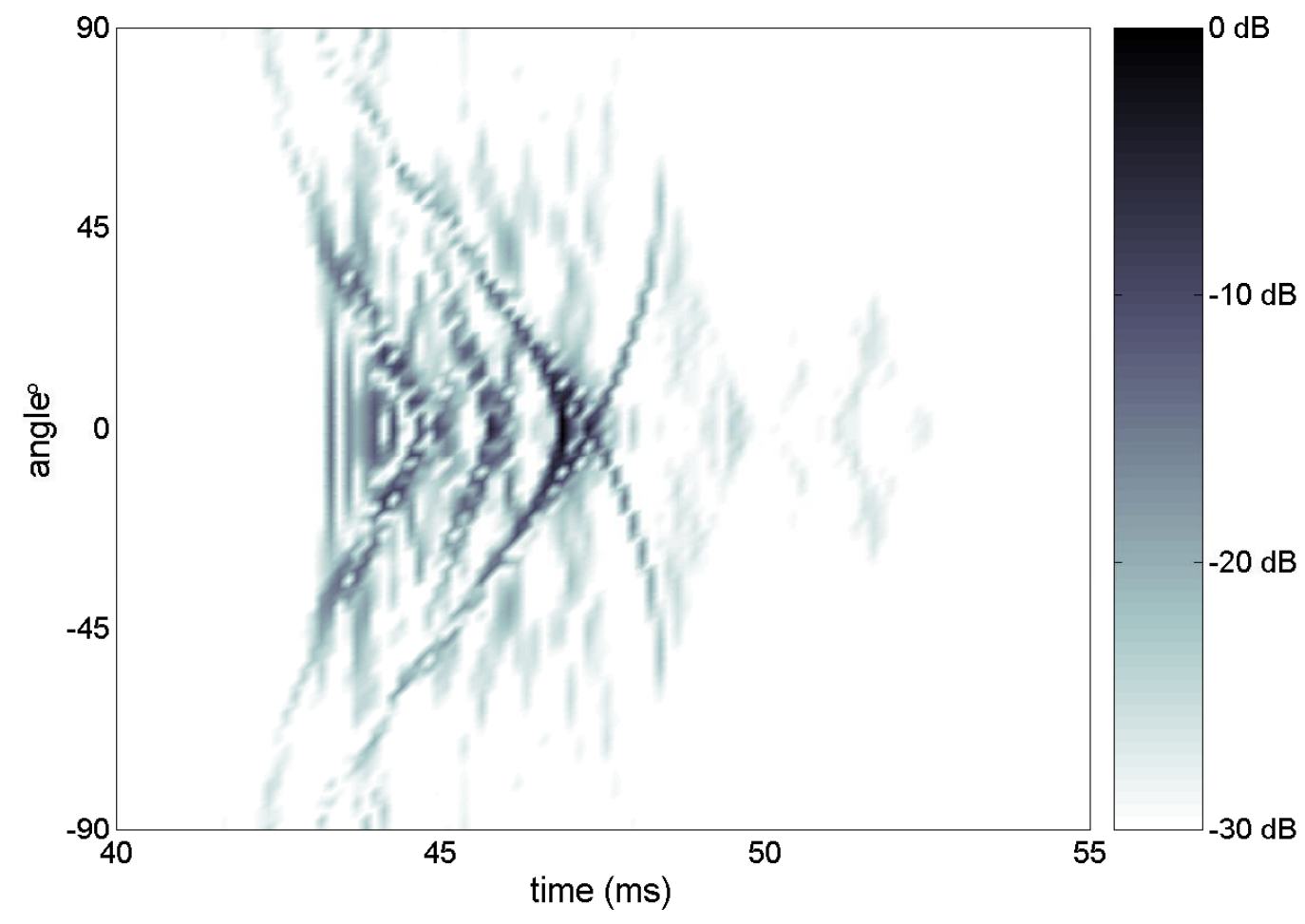

Figure 13 


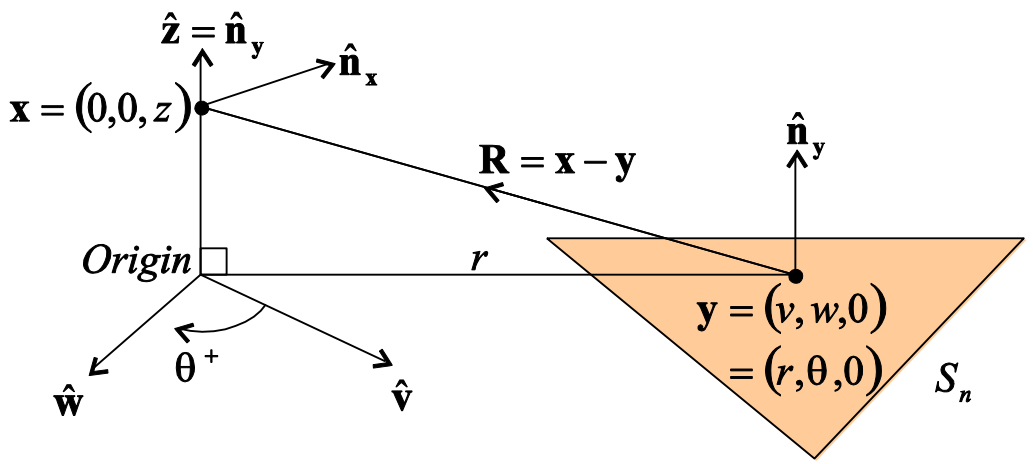

Figure 14 


\section{References}

1 M. B. Friedman and R. P. Shaw, "Diffraction of Pulses by Cylindrical Obstacles of Arbitrary Cross Section”, J. Appl. Mech. 29: 40 - 46 (1962)

2 A. J. Burton and G. F. Miller, "The application of integral equation methods to the numerical solution of some exterior boundary-value problems”, P. Roy. Soc. Lond. A. Mat. 323: $201-210(1971)$

3 A. A. Ergin, B. Shanker and E. Michielssen, “Analysis of transient wave scattering from rigid bodies using a Burton-Miller approach”, J. Acoust. Soc. Am. 106 (5): 2396 $-2404(1999)$

4 Y. Kawai and T. Terai, "A Numerical Method for the Calculation of Transient Acoustic Scattering from Thin Rigid Plates”, J. Sound Vib. 141 (1): 83 - 96 (1990)

5 A. A. Ergin, B. Shanker and E. Michielssen, "Fast Transient analysis of acoustic wave scattering from rigid bodies using a two-level plane wave time domain algorithm", J. Acoust. Soc. Am. 106 (5): 2405 - 2416 (1999)

6 A. A. Ergin, B. Shanker and E. Michielssen, "Fast analysis of transient acoustic wave scattering from rigid bodies using the multilevel plane wave time domain algorithm", J. Acoust. Soc. Am. 107 (3): 1168 - 1178 (2000) 
7 A. E. Yilmaz, J.-M. Jina and E. Michielssen, “A Fast Fourier Transform Accelerated Marching-on-in-Time Algorithm for Electromagnetic Analysis”, Electromagnetics 21: $181-197(2001)$

8 G. C. Herman and P. M. van den Berg, "A Least Squares Iteratively Technique for solving Time-Domain Scattering Problems”, J. Acoust. Soc. Am. 72 (6): 1947 - 1953 (1982)

9 Y. Shifman and Y. Leviatan, "On the Use of Spatio-Temporal Multiresolution Analysis in Method of Moments Solutions of Transient Electromagnetic Scattering", IEEE T. Antenn. Propag. 49 (8): 1123 - 1129 (2001)

10 Z. Ji, T. K. Sarkar, B. H. Jung, M. Yuan and M. Salazar-Palma, "Solving Time Domain Electric Field Integral Equation without the Time Variable”, IEEE T. Antenn. Propag. 54 (1): 258 - 262 (2006)

11 S. Barmada: "Improving the performance of the boundary element method with timedependent fundamental solutions by the use of a wavelet expansion in the time domain”, Int. J. Num. Meth. Eng. 71: 363 - 378 (2007)

12 A. D. Pierce, Acoustics: an introduction to its physical principles and application, McGraw-Hill 1981

13 J.-M. Parot, C. Thirard and C. Puillet, "Elimination of a non-oscillatory instability in a retarded potential integral equation”, Eng. Anal. Bound. Elem. 31: 133 - 151 (2007)

14 D. A. Vechinski and S. M. Rao, "Transient Scattering from dielectric cylinders: Efield, h-field and combined-field solutions", Radio Sci. 27 (5): 611 - 622 (1992) 
15 D. J. Chappell, P. J. Harris, D. Henwood and R. Chakrabarti, “A stable boundary integral equation method for modelling transient acoustic radiation”, J. Acoust. Soc. Am. 120 (1): 74 - 80 (2006)

16 R. Martinez, “The thin-shape breakdown (TSB) of the Helmholtz integral equation”, J. Acoust. Soc. Am. 90 (5): 2728 - 2738 (1991)

$17 \mathrm{~T} . \mathrm{W} . \mathrm{Wu}$, “A direct boundary element method for acoustic radiation and scattering from mixed regular and thin bodies", J. Acoust. Soc. Am. 97 (1): 84 - 91 (1995)

18 H. A. Schenck, "Improved integral formulation for acoustic radiation problems", J. Acoust. Soc. Am. 44 (1) $41-58$ (1968)

19 B. P. Rynne, "Instabilities in Time Marching Methods for Scattering Problems", Electromagnetics 6: $129-144$ (1986)

20 S. J. Dodson, S. P. Walker, M. J. Bluck, "Implicitness and stability of time domain integral equation scattering analysis", ACES. J. 13: 291 - 301 (1998)

21 P. D. Smith, "Instabilities in Time Marching Methods for Scattering: Cause and Rectification", Electromagnetics 10: 439 - 451 (1990)

22 H. Wang. D. J. Henwood, P. J. Harris and R. Chakrabarti, "Concerning the cause of instability in time-stepping boundary element methods applied to the exterior acoustic problem”, J.Sound.Vib. 305: 289 - 297 (2007)

23 B. P. Rynne and P. D. Smith, "Stability of Time Marching Algorithms for the Electric Field Integral Equation”, J. Electromagnet. Wave. 4 (12): 1181 - 1205 (1990) 
24 T. J. Cox and Y. W. Lam, "Evaluation of Methods for Predicting the Scattering from Simple Rigid Panels”, Appl. Acoust. 40: 123 - 140 (1993).

25 P D’Antonio and T J Cox, “Diffusor Application in Rooms”, Appl. Acoust. 60 (2): $113-142(2000)$

26 AES information document for room acoustics and sound reinforcement systems Characterisation and measurement of surface scattering uniformity. Audio Engineering Society (2001)

27 T. J. Cox, Y. W. Lam: "Prediction and Evaluation of the Scattering from Quadratic Residue Diffusers”, J. Acoust. Soc. Am. 95 (1) 297-305 (1994)

28 T. J. Cox and P. D’Antonio, Acoustic Absorbers and Diffusers, Spon Press (2004)

29 M. R. Schroeder, "Diffuse sound reflection by maximum-length sequences”, J. Acoust. Soc. Am. 57 (1): 149 - 150 (1975)

30 M. R. Schroeder, "Binaural dissimilarity and optimum ceilings for concert halls: More lateral sound diffusion”, J. Acoust. Soc. Am. 65 (4): 958 - 963 (1979)

31 A. Farina: "A new method for measuring the scattering coefficient and the diffusion coefficient of panels” Acustica 86 (6): 928 - 942 (2000)

32 J. Redondo, R. Picó, B. Roig and M. R. Avis: "Time Domain Simulation of Sound Diffusers Using Finite-Difference Schemes”, Acustica 93 (4) 611 - 622 (2007) 
33 J. A. Hargreaves and T. J. Cox, “A transient boundary element method model of Schroeder diffuser scattering using well mouth impedance", J.Acoust.Soc.Am. 124 (5): $2942-2951(2008)$

34 T. Ha-Duong, B. Ludwig and I.Terrasse, "A Galerkin BEM for transient acoustic scattering by an absorbing obstacle”, Int. J. Numer. Meth. Engng. 57: 1845 - 1882 (2003) 


\section{Figure Captions}

Figure 1: A scattering problem comprising an obstacle submerged in a connected medium. $S$ is a surface conformal to the obstacle, hence the medium is said to be external to $S$.

Figure 2: Cross-section of a thin body section

Figure 3: The cube plus fin mesh

Figure 4: Scattered Pressure in Pascals for two receivers, one inside and one outside the body of the cube plus fin mixed model, excited by a $0.02 \mathrm{~s}$ Hanning plane wave.

Figure 5: Scattered Pressure in $\mathrm{dB}$ for two receivers, one inside and one outside the body of the cube plus fin mixed model, excited by a $0.02 \mathrm{~s}$ Hanning plane wave.

Figure 6: Discrete poles of the cube plus fin mixed model

Figure 7: Scattered Pressure in $\mathrm{dB}$ for two receivers, one inside and one outside the body of the cube plus fin all-thin model, excited by a 0.02 s Hanning plane wave.

Figure 8: Discrete poles of the cube plus fin all-thin model

Figure 9: The Quadratic Residue Diffuser (QRD) Mesh

Figure 10: Discrete poles of the QRD mixed model 
ACTA ACUSTICA UNITED WITH ACUSTICA Vol. 95 (2009) 678 - 689 DOI 10.3813/AAA.918196

Figure 11: Scattered Pressure in $\mathrm{dB}$ for two receivers, one inside and one outside the body of the QRD mixed model, excited by a 0.02s Hanning plane wave.

Figure 12: Scattered Pressure in $\mathrm{dB}$ for two receivers, one inside and one outside the body of the QRD all-thin model, excited by a 0.02s Hanning plane wave

Figure 13: Normalised transient scattered velocity potential at a $5 \mathrm{~m}$ radius receiver arc

Figure 14: Contour integration geometry and coordinate systems 